\title{
Endoscopic Treatment of an Adult with Tegmental Astrocytoma Accompanied by Cerebrospinal Fluid Dissemination
}

\author{
Runchun Lu, M.D., ${ }^{1}$ Chuzhong Li, M.D., ${ }^{2}$ Xinsheng Wang, M.D., ${ }^{2}$ Yazhuo Zhang, M.D. ${ }^{2}$ \\ Neurosurgical Department, ${ }^{1}$ Peking University First Hospital, Beijing, China \\ Beijing Neurosurgical Institute, ${ }^{2}$ Beijing, China
}

\begin{abstract}
Midbrain gliomas are relatively rare neoplasms with a generally benign prognosis, with dissemination or metastasis not previously reported. We describe here a woman, in whom magnetic resonance imaging scans showed hydrocephalus and a tegmental lesion in the upper aqueduct. Endoscopic third ventriculostomy and biopsy were performed; during surgery, a second small lesion was observed in the infundibular recess. Histologically, the two lesions had the characteristics of low grade astrocytoma, suggesting that the midbrain astrocytoma may have been disseminated via the cerebral spinal fluid to the infundibular recess. Postoperatively this patient received radiotherapy for nearly one month. Although patients with these tumors are not usually administered adjunctive therapy, radiation and, combined modality therapy, including surgery, radiotherapy, and chemotherapy, may be beneficial in patients with midbrain gliomas with dissemination.
\end{abstract}

Key Words : Neuroendoscope · Astrocytoma · Tegmentum · Dissemination · Biopsy.

\section{INTRODUCTION}

Tumors of the midbrain often cause obstruction of the cerebral aqueduct, leading to hydrocephalus. Endoscopic ventriculostomy and biopsy are considered suitable operations for these patients ${ }^{13}$. Astrocytomas in the peri-aqueductal area are usually low grade and manifest relatively benign growth characteristics, both of which suggest a good prognosis ${ }^{9,10,12)}$. Although some low grade astrocytomas can present with atypical behavior, characterized by metastatic spread $d^{1,2,4-6,8,11,14,16,17,20)}$, all of those tumors were low grade gliomas located in the posterior fossa, with most being pilocytic astrocytomas in chil- dren. We describe here an adult with a low grade astrocytoma in the third ventricle (tegmentum), associated with ventricular dissemination and hydrocephalus, who was treated endoscopically. We discuss treatment and the possible mechanism of metastasis.

\section{CASE REPORT}

A 58-year-old female presented with headache, nausea, vomiting, and visual impairment lasting for one year. Neurological examination showed bilateral papilledema. There was

- Received : August 22, 2013 •Revised : July 28, 2013 •Accepted : October 12, 2014

- Address for reprints : Yazhuo Zhang, M.D.

Beijing Neurosurgical Institute, 6 TiantanXili, Dongcheng District, Beijing 100050, China

Tel : +86-(0)10-67096763, Fax : +86-(0)10-67057391, E-mail : zyz2004520@yeah.net

This is an Open Access article distributed under the terms of the Creative Commons Attribution Non-Commercial License (http://creativecommons.org/licenses/by-nc/4.0) which permits unrestricted non-commercial use, distribution, and reproduction in any medium, provided the original work is properly cited. 
no obvious memory loss, instability of gait, or urinary incontinence. Cranial magnetic resonance imaging (MRI) showed an obstructive hydrocephalus and a bulky lesion in the tegmentum close to the upper aqueduct, with a hypointense signal on T1-weighted MRI without enhancement (Fig. 1A-E). We performed an endoscopic third ventriculostomy and biopsy (Karl Storz, Tuttlingen, Germany). We choose a left frontal craniotomy, because the right frontal angle and interventricular foramen could not be dilated sufficiently to insert the endoscope (Fig. 1F). After introduction of the endoscope into the third ventricle, we observed a grey lesion located in the infundibular recess (Fig. 2A), not observed on the MRI (Fig. $1 C, D)$. A biopsy was taken, showing that the tumor had a poor blood supply and obscure boundaries (Fig. 2B). A third ventriculostomy was performed to achieve cerebrospinal fluid (CSF) flow between the ventricle and the cisterns (Fig. 2C). A grey, lobulated lesion, larger than the region in the infundibular recess, was observed in the upper aqueduct (Fig. 2D, E). Endoscopic biopsy showed that this tumor had a poor blood supply and adhered to the brain stem (Fig. 2F). The tumor was resected endoscopically as much as possible, although the aqueduct could not be completely cleared out. We also performed a fenestration of the septum pellucidum and confirmed that the contralateral interventricular foramen was normal and without obstruction (Fig. 2G, H).

Following the operation, the patient's symptoms, including headache, nausea, and visual impairment, were improved. An MRI performed two days later showed that the lesion in the upper aqueduct had not been completely removed (Fig. 1G, $\mathrm{H})$. Histological examinations of the two lesions both showed that both were low grade astrocytomas with Rosenthal fibers (Fig. 3). Because of the lack of adequate tumor tissue for complete histological examination, however, the exact pathological type (world health organization [WHO] 2007) of the tumor could not be determined.

One month after surgery, the patient was started on radiotherapy of the brain, lasting for 25 days, since tumor dissemination may suggest a more aggressive pathological behavior. Her recovery has been uneventful, with no further neurological deterioration
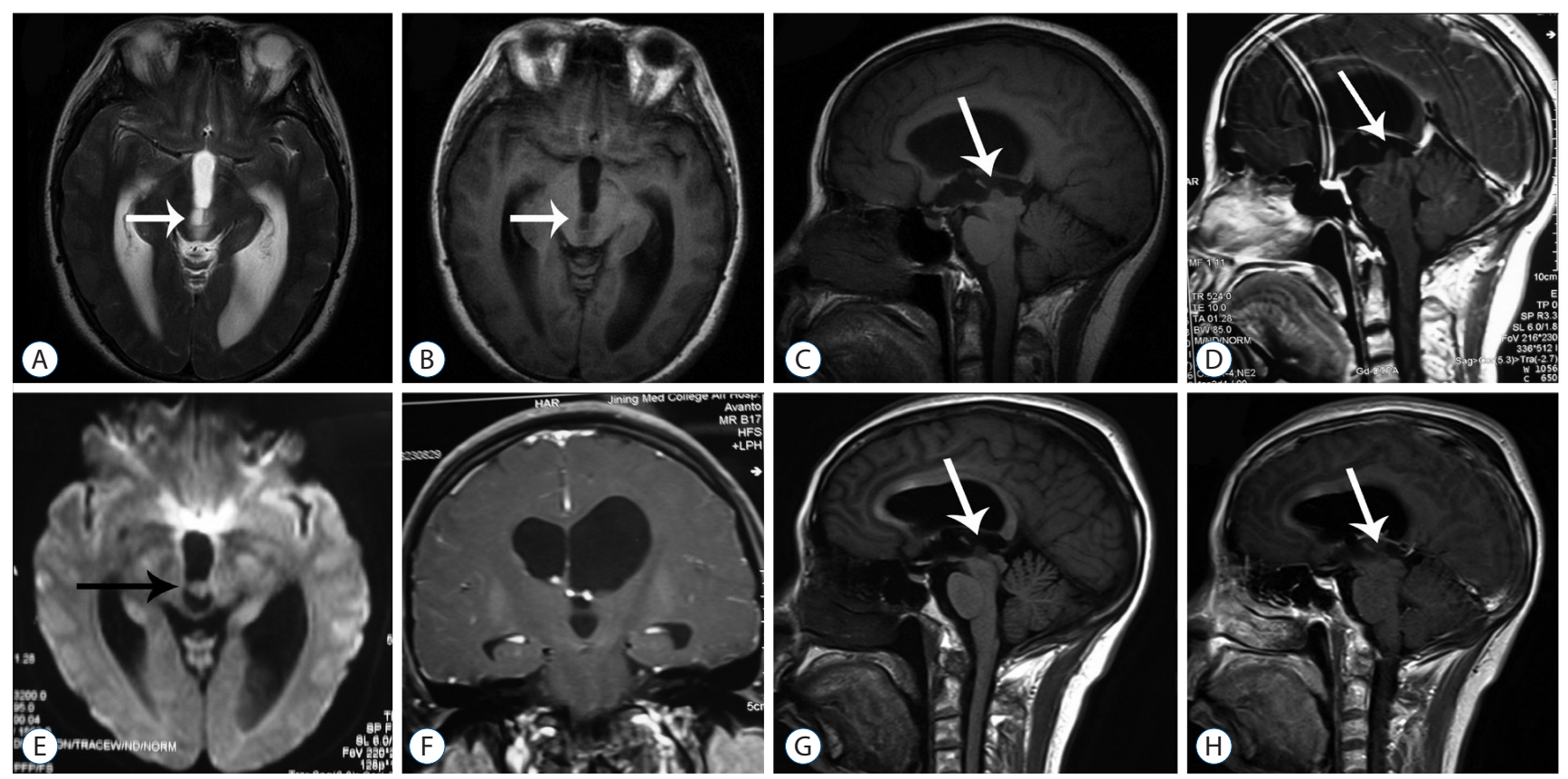

Fig. 1. Magnetic resonance (MR) imaging in our patient. A : Axial T2 weighted MR image showing a lesion in the dorsal midbrain (arrow) and enlargement of the ventricles. B : Axial T1 weighted MR image showing a lesion in the dorsal midbrain (arrow). C : Sagittal T1 weighted MR image showing a lesion in the tegmentum (arrow) resulting in obstruction of the aqueduct. D : Sagittal T1 weighted MR image with gadolinium showing a lesion in the tegmentum (arrow). E : Diffuse weighted imaging showing a lesion in the third ventricle (arrow). F : Coronal T1 weighted MR image showing that the left lateral ventricle is larger than the right. G : Postoperative sagittal T1 weighted MR image showing the tumor in the tegmentum (arrow). $\mathrm{H}$ : Postoperative sagittal T1 weighted MR image with gadolinium showing the tumor in the tegmentum (arrow). 

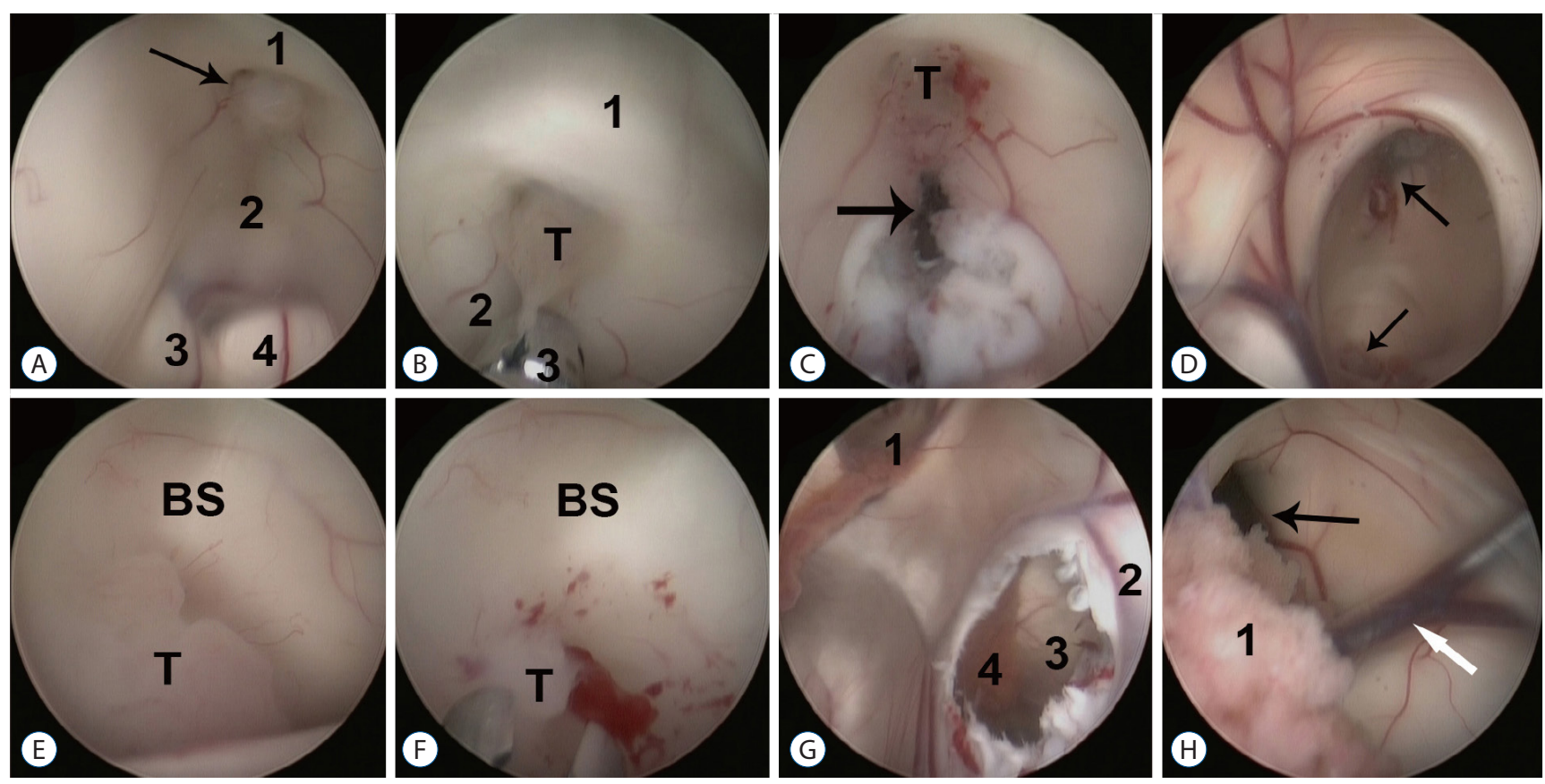

Fig. 2. Endoscopic findings in our patient. A : Image showing a neoplasm (arrow) in the fundibular recess between the lamina terminalis (1) and the floor of the third ventricle (2); 3 and 4 are the right and left cerebral peduncles, respectively. B : Biopsy of the tumor (T) using a microforceps (3). The lamina terminalis (1) and the floor of the third ventricle (2) were anterior and posterior to the tumor, respectively. C : Endoscopic fenestration (arrow) near the tumor (T). D : Obstruction of the aqueduct by a lesion (bottom arrow); the top arrow indicates the position of the third ventriculostomy. $E$ : Close-up view of the tumor (T) in the brainstem (T). F : biopsy of the tumor (T) using a microforceps. $G$ : Endoscopic view of the left lateral ventricle; the left interventricular foramen (1) was confirmed. Fenestration (3) of the septum pellucidum (4) was performed, making the contralateral choroid plexus (4) visible. $\mathrm{H}$ : Endoscopic view of the right lateral ventricle following fenestration of the septum pellucidum, the choroid plexus in the right ventricle (1), the right interventricular foramen (black arrow), and the vena thalamostriata (white arrow). BS : brain stem.

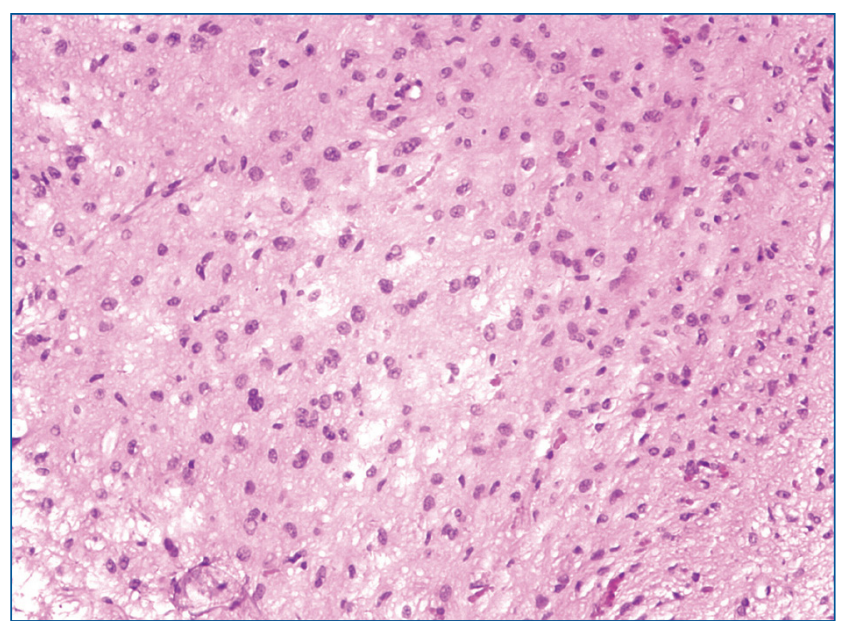

Fig. 3. Histological examination of the tumor tissue following hematoxylin and eosin staining $(H \& E, \times 200)$.

\section{DISCUSSION}

Brain stem tumors are relatively uncommon, accounting for about $2 \%$ of the intracranial tumors treated in our institution, with primary midbrain gliomas accounting for about $10 \%$ of brain stem tumors ${ }^{21}$. Most of these tumors are low grade gliomas with a relatively benign prognosis. Adult patients have better outcomes than children. Although disseminated low grade gliomas have been reported previously, midbrain gliomas with dissemination have, to our knowledge, not been described. We report the first case of a tegmental low grade astrocytoma accompanied by ventricular dissemination, which was detected during endoscopy but not during cranial imaging.

Most tumors in the tegmental area are low grade astrocyto$\mathrm{mas}^{21}$. Although the specific pathological type (world health organization [WHO] 2007) of this tumor could not be determined because of a lack of sufficient tumor tissue, pathological examination revealed a low grade astrocytoma. Both tu- 
mors in our patient had similar histological characteristics, with the lesion in the tegmentum being larger than the lesion in the infundibular recess, suggesting that the smaller lesion is likely a secondary tumor derived from the larger one. Since both lesions were located in the ventricles, dissemination likely occurred through CSF flow. Dissemination of low grade gliomas through the CSF even shunt has been reported previous$1 y^{2,4,5,14-16)}$. Other metastatic patterns have also been reported, including leptomeningeal spread and white matter tract trans$\mathrm{fer}^{4,5,8,11)}$. The molecular mechanisms underlying the metastasis of low grade astrocytomas have not been fully elucidated, although they may involve specific extracellular substrates and metabolic capacities.

Patients with focal midbrain gliomas require treatment of hydrocephalus resulting from tumor obstruction of the aqueduct. Aggressive treatment methods, such as surgical debulking, radiation therapy or chemotherapy, are often not required $^{7)}$, with only one study recommending surgery ${ }^{21}$. Although adults with brain stem gliomas have a better prognosis than children ${ }^{18)}$, there is insufficient data on midbrain gliomas to demonstrate a difference between these two age groups. Adults and children with focal midbrain gliomas should be treated similarly ${ }^{23)}$, with aggressive treatments, such as tumor resection, radiotherapy, and chemotherapy, generally not recommended. It remains unclear, however, whether patients with dissemination at diagnosis require more aggressive treatments. We treated our patient by biopsy and treatment of the obstructive hydrocephalus. Since ventricular dissemination was confirmed both endoscopically and histologically, we included adjunctive radiotherapy to enhance quality of life. Both V-P shunts and ETV are options for the treatment of hydrocephalus in those patients ${ }^{10,13,22,23)}$. However endoscopic surgery has been recommended for patients with midbrain gliomas for several reasons : 1) these patients present with obstructive hydrocephalus, indicating the need for ETV; 2) the tumor can be at least partially resected during endoscopic biopsy; and 3) surgery can avoid shunt-related complications. Endoscopic surgery is also the treatment of choice for patients with focal midbrain gliomas accompanied by hydrocephalus ${ }^{3,10,13,19)}$, with some requiring surgical resection $^{211}$.

\section{CONCLUSION}

Patients with midbrain gliomas usually accompanied with hydrocephalus. Those patients mostly presented with hydrocephalys related symptoms, such as headache, vomiting, etc. Endoscopic third ventriculostomy, which can resolve hydrocephalus as well as get biopsy or resection of the tumor, is a suitable choice for those patients. Radiotherapy is opt for certain patients. Dissemination, which mainly through CSF, is rare in midbrain gliomas. More aggressive treatment maybe needed for Disseminated cases. Endoscopic surgery is a effective therapy for those accompanied with ventricular dissemination.

\section{References}

1. Andrychowski J, Taraszewska A, Czernicki Z, Jurkiewicz J, Netczuk T, Dabrowski $P$ : Ten years observation and treatment of multifocal pilocytic astrocytoma. Folia Neuropathol 47 : 362-370, 2009

2. Buschmann U, Gers B, Hildebrandt G : Pilocytic astrocytomas with leptomeningeal dissemination : biological behavior, clinical course, and therapeutical options. Childs Nerv Syst 19 : 298-304, 2003

3. Domínguez-Páez M, Puch-Ramírez M, Rodríguez-Barceló S, Medina-Imbroda JM, Romero-Moreno L, Ibáñez-Botella G, et al. : Neuroendoscopic biopsy. Experience in 31 patients and literature review. Neurocirugia (Astur) 22 : 419-427; discussion 428, 2011

4. Faria AV, Azevedo GC, Zanardi VA, Ghizoni E, Queiroz LS : Dissemination patterns of pilocytic astrocytoma. Clin Neurol Neurosurg 108 : 568572, 2006

5. Figueiredo EG, Matushita H, Machado AG, Plese JP, Rosemberg S, Marino $\mathrm{R} \mathrm{Jr}$ : Leptomeningeal dissemination of pilocytic astrocytoma at diagnosis in childhood: two cases report. Arq Neuropsiquiatr 61(3B) : 842847, 2003

6. Gajjar A, Bhargava R, Jenkins JJ, Heideman R, Sanford RA, Langston JW, et al. : Low-grade astrocytoma with neuraxis dissemination at diagnosis. J Neurosurg 83 : 67-71, 1995

7. Hamilton MG, Lauryssen $C$, Hagen $N$ : Focal midbrain glioma : long term survival in a cohort of 16 patients and the implications for management. Can J Neurol Sci 23 : 204-207, 1996

8. Jamjoom AB, Jamjoom ZA, al-Rayess $M$ : Intraventricular and leptomeningeal dissemination of a pilocytic cerebellar astrocytoma in a child with a ventriculoperitoneal shunt : case report. Br J Neurosurg 12 : 56-58, 1998

9. Lázaro BC, Landeiro JA : Tectal plate tumors. Arq Neuropsiquiatr 64 (2B) : 432-436, 2006

10. Li KW, Roonprapunt C, Lawson HC, Abbott IR, Wisoff J, Epstein F, et al. : Endoscopic third ventriculostomy for hydrocephalus associated with 
tectal gliomas. Neurosurg Focus 18(6A) : E2, 2005

11. Moon JH, Jung TY, Jung $\mathrm{S}$, Jang $\mathrm{WY}$ : Leptomeningeal dissemination of a low-grade brainstem glioma without local recurrence. J Korean Neurosurg Soc 51 : 109-112, 2012

12. Oka K, Kin Y, Go Y, Ueno Y, Hirakawa K, Tomonaga M, et al. : Neuroendoscopic approach to tectal tumors : a consecutive series. J Neurosurg 91 : 964-970, 1999

13. Oppido PA, Fiorindi A, Benvenuti L, Cattani F, Cipri S, Gangemi M, et al. : Neuroendoscopic biopsy of ventricular tumors : a multicentric experience. Neurosurg Focus 30 : E2, 2011

14. Pollack IF, Hurtt M, Pang D, Albright AL : Dissemination of low grade intracranial astrocytomas in children. Cancer 73 : 2869-2878, 1994

15. Redhu R, Nadkarni TD : Multicompartmental cerebrospinal fluid dissemination of cerebellar pilocytic astrocytoma at presentation. J Clin Neurosci 18 : 1412-1414, 2011

16. Sakai K, Miyahara T, Tsutsumi K, Kaneko T, Fukushima M, Tanaka Y, et al. : Spontaneous regression of multicentric pilocytic astrocytoma with CSF dissemination in an adult. Brain Tumor Pathol 28 : 151-156, 2011

17. Salvati M, Oppido PA, Artizzu S, Fiorenza F, Puzzilli F, Orlando ER : Multicentric gliomas. Report of seven cases. Tumori 77 : 518-522, 1991
18. Selvapandian S, Rajshekhar V, Chandy MJ : Brainstem glioma : comparative study of clinico-radiological presentation, pathology and outcome in children and adults. Acta Neurochir (Wien) 141 : 721-726; discussion 726-727, 1999

19. Tirakotai W, Hellwig D, Bertalanffy $H$, Riegel $T$ : The role of neuroendoscopy in the management of solid or solid-cystic intra- and periventricular tumours. Childs Nerv Syst 23 : 653-658, 2007

20. Uro-Coste E, Ssi-Yan-Kai G, Guilbeau-Frugier C, Boetto S, Bertozzi Al, Sevely $A$, et al. : Desmoplastic infantile astrocytoma with benign histological phenotype and multiple intracranial localizations at presentation. J Neurooncol 98 : 143-149, 2010

21. Wang C, Zhang J, Liu A, Sun B, Zhao Y : Surgical treatment of primary midbrain gliomas. Surg Neurol $53:$ 41-51, 2000

22. Wellons JC 3rd, Tubbs RS, Banks JT, Grabb B, Blount JP, Oakes WJ, et al. : Long-term control of hydrocephalus via endoscopic third ventriculostomy in children with tectal plate gliomas. Neurosurgery 51 : 6367; discussion 67-68, 2002

23. Yeh DD, Warnick RE, Ernst RJ : Management strategy for adult patients with dorsal midbrain gliomas. Neurosurgery 50 : 735-738; discussion 738-740, 2002 